\section{Climica Ciriongica \\ A VIDEOLAPAROSCOPIA NA HÉRNIA INGUINAL DEVE SER ROTINEIRA?}

As supostas vantagens da hernioplastia videolaparoscópica sobre a técnica convencional, até o momento, não foram consistentemente demonstradas, tanto que, nos Estados Unidos da América, somente 13\% das correções de hérnias inguinais são feitas por este método ${ }^{6}$.

Alguns aspectos podem ser citados para tentar explicar a aceitação limitada desta técnica: a relativa facilidade de realizar reparos abertos sem tensão, até mesmo com anestesia local e curta permanência hospitalar; índices de recidiva e de complicações bastante reduzidos na cirurgia convencional; maior custo e necessidade de equipamentos mais complexos para a realização da técnica laparoscópica; maior potencial de complicações relacionadas ao procedimento laparoscópico, ao pneumoperitôneo e à anestesia geral; maior familiaridade do cirurgião com a anatomia da região inguinal pela via anterior; e o uso rotineiro e obrigatório de prótese em todos os casos pela via laparoscópica ${ }^{6}$.

Apesar disso, duas vantagens relacionadas ao método laparoscópico são citadas e amplamente aceitas na literatura: quase ausência de dor pós-operatória; maior facilidade na dissecção anatômica de hérnias recidivadas, tratadas previamente pelo acesso anterior; e um tempo de retorno mais precoce às atividades habituais e ao trabalho ${ }^{5}$.

Outro aspecto que deve ser discutido é com relação à curva de aprendizado excessivamente longa na técnica videolaparoscópica, sendo que muitos autores relacionam os índices de complicações à inexperiência dos cirurgiões ${ }^{2,3}$. Entretanto, muitos dos trabalhos que mostram taxas de complicações pela técnica laparoscópica inferiores à aberta não discriminam com precisão os grupos de pacientes estudados e o tipo de hérnia que eles apresentavam ${ }^{4}$.

que se nota com clareza, na literatura, é que as complicações intra-operatórias da correção laparoscópica, quando ocorrem, são significativamente mais graves do que as complicações encontradas nos reparos abertos. Podemos destacar as lesões provocadas pelos trocarteres, e pela clipagem de nervos e vasos!.

Dessa forma, até o presente momento, não há dados na literatura que possam recomendar o emprego rotineiro da hernioplastia inguinal videolaparoscópica.

Osvaldo Antonio Prado Castro RODRIGO VincenzI hassan Fahd El Malat ELIAS JiRJoss ILIAS Paulo Kassab

Referências

I.Bendavid R. Complications of groin hernia surgery Surg Clin North Am 1998;78:1089-103.
2.DeTurris SV, Cacchione RN, Mungara A, Pecoraro A, Ferzli GS. Laparoscopic herniorrhaphy: beyond the learning curve. J Am Coll Surg 2002; 194:65-73.

3.Felix E, Scott S, Crafton B, Geis P, Duncan T, Sewell R, et al. Causes of recurrence after laparoscopic hernioplasty: multicenter study. Surg Endosc | 998; | 2:226-3|.

4.Liem MS, Van der Graaf Y, Van Steensel C], Boelhouwer RU, Clevers G], MeijerWS, et al. Comparison of conventional anterior surgery and laparoscopic surgery for inguinal-hernia repair. N Engl J Med 1997;336: I 54 I-7.

5.Oberlin P. Faut-il opérer toutes les hernies de láine? Ann Chir 2002; 127: $161-3$.

6.Safadi BY, Duh QY. Minimally invasive approaches to inguinal hernia repair. J Laparoendosc Adv Surg Tech A 200 I ; I 1:36 I -6.

\section{Emergênela e Mediceuna Intendiua No QUE CONSISTE A MONITORIZAÇÃO NEUROLÓGICA À BEIRA DO LEITO?}

A monitorização do paciente neurológico grave na UTI consiste em identificar, por meio do exame físico e dos equipamentos monitores invasivos sinais, que indiquem hipertensão intracraniana e diminuição de perfusão cerebral, que se não forem revertidas levam à morte cerebral.

A pressão intracraniana normal é $4 \mathrm{mmHg}$. Em situações de injúria cerebral como trauma ou isquemia, a maioria dos autores aceita como limite superior de pressão o valor de $20 \mathrm{mmHg}$. Valores acima disto podem levar a edemas com herniação, que é a protrusão de tecido cerebral através das tendas cerebrais (dobras de membrana de dura-mater que servem de sustentação ao parênquima cerebral). A herniação comprime tecidos e vasos adjacentes, podendo interromper a circulação local, levando à necrose. $O$ tronco é área particularmente nobre, por regular a pressão, temperatura e freqüências respiratória e cardíaca e por conter o sistema reticular ativador ascendente, que regula o despertar e a capacidade de interação com o meio ambiente. É fundamental no paciente em coma determinar o grau de sofrimento do tronco.

A monitorização se inicia por um exame neurológico sucinto no qual se avaliam sinais que indicam compressão do tronco ou dos nervos cranianos adjacentes ao mesmo. Avaliamos o tamanho e a simetria das pupilas, bem como a sua reação à luz, que permitem inferir sobre compressões do terceiro e quarto nervos. Pupilas dilatadas e arreativas indicam lesão bilateral do tronco. Assimetria (anisocoria) indica lesão unilateral de tronco por herniação de porção do lobo temporal (uncus). A presença de posturas motoras anômalas decorticação (flexão de membros superiores e extensão de membros inferiores) e descerebração (extensão de membros superiores e inferiores) indica progressiva lesão de tronco. Estes sinais aparecem tardiamente, sendo insuficientes para permitir bons resultados.

A pressão intracraniana (PIC) pode ser medida diretamente por meio da introdução intracerebral de cateteres 
permeados com fluido ou de fibra óptica ligados a transdutor que permita medidas contínuas da PIC. O cateter permeado com fluido é barato e sensível, sendo colocado no ventrículo lateral, o que permite lenta drenagem de líquor, se a PIC for alta. Pode ser recalibrado. Apresenta alto índice de infecção, variando na literatura entre $7 \%$ e 40\%. O cateter de fibra óptica pode ser deixado em parênquima ou ventrículo lateral, sendo sensível, porém mais caro e não permitindo drenagem de líquor nem recalibração. Os cateteres de PIC devem ser usados por no máximo cinco dias, sendo trocados se necessário.

O cateter no trauma de crânio está indicado se a tomografia computadorizada (TC) de crânio for alterada (presença de hematoma, contusão, edema ou compressão de cisternas basais) ou se a TC for normal e tivermos a presença de dois de três sinais propedêuticos (idade acima de 40 anos, PA sistólica $<90 \mathrm{mmHg}$ ou postura motora anômala).

A PIC deve ser mantida abaixo de $20 \mathrm{mmHg}$ por meio de sedação com propofol ou dormonid associados a fentanyl, hiperventilação leve ( $\mathrm{pCO}_{2}$ em torno de $35 \mathrm{mmHg}$ ), uso de manitol $(0,25-1,0 \mathrm{gr} / \mathrm{kg})$ em bolus. Medidas adicionais como hiperventilação entre $30-50 \mathrm{mmHg}$ ou sedação com barbitúricos solicitam o uso de monitorização metabólica.

A mensuração contínua da saturação de oxigênio por meio de cateter inserido na veia jugular, mantido na altura do bulbo, permite avaliar situações de isquemia em grande porção do hemisfério escolhido. Saturações abaixo de 55\% indicam que o tecido está ávido por oxigênio, indicando isquemia de causa global (hipóxia, hipotensão ou anemia) ou cerebral (herniação).

A limitação do método é que, às vezes, fatores regionais podem ser ignorados (vasoespasmo, isquemia de pequeno segmento cerebral, contusão focal). Um monitor do tipo Vigilance produzido pela Edwards é necessário. O cateter tem diâmetro 4F (muito fino), sendo fixado com cuidado. Deve ser trocado após cinco dias.

A mensuração da tensão de oxigênio por meio de cateter intraparenquimatoso avalia a saturação da hemoglobina tecidual (valor normal de $40 \mathrm{mmHg}$ ), sendo necessário um monitor multimodal (Neurotrend produzido pela Camino). É um método sensível, podemos usar mais de um cateter em áreas íntegras e lesadas para comparação. É um excelente método para isquemia regional (AVCi, vasoespasmo, contusões pequenas). Pode ser usado no intra-operatório de cirurgia de aneurisma com clipagem temporária.

A microdiálise é um método que consiste na passagem intraparenquimatosa de um cateter de fino diâmetro $(0,6 \mathrm{~mm})$, que permite que seja infundida continuamente uma solução de ringer lactato, reabsorvida pelo próprio cateter. $\bigcirc$ líquido permite a coleta de lactato, piruvato, glicerol, glicose e glutamato. Uma relação lactato/piruvato aumentada (valor normal $23+/-4$ ) indica isquemia tecidual.
Portanto, a avaliação do doente neurológico crítico se dá por exame neurológico associado a medidas de pressão e metabólicas.

IOANNIS M. LIONTAKIS

Referências

I. De Georgia MA, Deongaokar A. Multimodal monitoring in the neurological intensive care unit. Neurologist 2005; I I ( I):45-54.

2. Vincent JL, Berré J. Primer on medical management of severe brain injury. Crit Care Med 2005;33(6): | 392-9.

\section{Ginecologia}

\section{A VIA DE ACESSO PARA 0 IMPLANTE MAMÁRIO DE SILICONE PODE ALTERAR A PESQUISA DO LINFONODO SENTINELA EM EVENTUAL CÂNCER DE MAMA?}

A mastoplastia de aumento é um procedimento cirúrgico estabelecido e se constitui na primeira opção do tratamento das hipomastias!

Com o aprimoramento técnico, novas opções de acesso cirúrgico surgiram, destacando-se a axilar, que redundou em resultados estéticos mais favoráveis'. Entretanto, a despeito da maior indicação nos últimos anos, a incisão axilar não é isenta de riscos e atualmente são relatados questionamentos quanto à interrupção da drenagem linfática mamária',2 .

$\bigcirc$ principal deles considera que, na eventualidade da paciente desenvolver câncer de mama, a presença de cicatrizes no trajeto da drenagem linfática poderia alterar a anatomia normal, prejudicando, dessa forma, a identificação do linfonodo sentinela ${ }^{1,2}$. Daí a importância da preservação da drenagem que não interferiria no tratamento da paciente, uma vez que o comprometimento axilar pela neoplasia representa importante elemento da terapia adjuvante ${ }^{2,3}$.

Apesar do raciocínio lógico, não há até o presente momento estudos clínicos que comparem a identificação do linfonodo sentinela em pacientes com e sem implantes colocados pela via axilar'. No entanto, outros estudos que pesquisaram linfonodo sentinela em situações mais agressivas, como a biópsia prévia a céu aberto e por agulha fina, não observaram dificuldades técnicas ${ }^{4}$, permitindo-nos supor sobre a não influência na alteração da pesquisa do linfonodo sentinela.

Munhoz et al.' ', ao descreverem a linfocintilografia comparativa antes e após a colocação da prótese por via axilar, também não observaram alterações na localização do linfonodo sentinela.

Assim, em face da inexistência de estudos pertinentes ao tema e das controvérsias pendentes, tornam-se imperiosas investigações randomizadas, placebo controladas e prospectivas no tocante às diferentes vias de acesso e à acurácia na detecção do linfonodo sentinela. 